\title{
Violência obstétrica: perspectiva de puérperas atendidas em um hospital universitário no interior de Minas Gerais
}

Obstetric violence: perspective of postpartum women attended in a university hospital inside Minas Gerais

Violencia obstétrica: perspectiva de mujeres posparto asistidas en un hospital universitario dentro de Minas Gerais

\begin{abstract}
RESUMO
Objetivo: Analisar o quanto a implementação do projeto Apice On contribuiu para a redução de casos de violência obstétrica vivenciadas por puérperas atendidas em um Hospital Universitário no interior de Minas Gerais. Método: Estudo exploratório, descritivo, transversal e de caráter qualitativo, desenvolvido com 46 puérperas atendidas em um Hospital Universitário. Os dados foram coletados por meio de um instrumento semiestruturado, os quais foram analisados posteriormente utilizando a técnica de Bardin e o uso do software Iramuteq. Resultados: As puérperas entrevistadas são empoderadas frente aos seus direitos, reconhecem as possiveis faces da violência obstétrica, e possuem preferência pelo parto normal humanizado, tendo conhecimento sobre a abordagem correta do atendimento profissional. Conclusão: Conclui-se que a implementação do projeto Apice On e da enfermeira obstétrica contribuiu para a redução de casos de violência obstétrica e cumpriu com seus objetivos frente a humanização dos partos normais realizados no Hospital Universitário do interior de Minas Gerais.
\end{abstract}

DESCRITORES: Enfermeira Obstétrica; Parto Normal; Violência contra a mulher.

\section{ABSTRACT}

Objective: Analyze how the implementation of the Apice On program contributed to reduce the cases of obstetric violence experienced by postpartum women attended at the a University Hospital in the interior of Minas Gerais. Method: Exploratory, descriptive, cross-sectional and qualitative study, developed with 46 postpartum women attended at a University Hospital. Data were collected using a semi-structured instrument, which were subsequently analyzed using the Bardin technique, in addition to the use of the Iramuteq software. Results: The interviewed puerperal women are empowered in the face of their rights, recognize the possible faces of obstetric violence, and have a preference for normal humanized delivery, having knowledge about the correct approach to professional care. Conclusion: It is concluded that the implementation of the Apice On project and the obstetric nurse contributed to the reduction of cases of obstetric violence and fulfilled its objectives in view of the humanization of normal births performed at the University Hospital in the interior of Minas Gerais.

DESCRIPTORS: Obstetric Nurse; Normal Childbirth; Violence against a woman.

\section{RESUMEN}

Objetivo: Analizar en qué medida la implementación del proyecto Apice On contribuyó a la reducción de los casos de violencia obstétrica vividos por puérperas atendidas en un Hospital Universitario del interior de Minas Gerais. Método: Estudio exploratorio, descriptivo, transversal y cualitativo, desarrollado con 46 puérperas atendidas en un Hospital Universitario. Los datos se recogieron mediante un instrumento semiestructurado, que posteriormente se analizaron mediante la técnica de Bardin, además del uso del software Iramuteq. Resultados: Las madres entrevistadas se encuentran empoderadas frente a sus derechos, reconocen los posibles rostros de la violencia obstétrica y tienen preferencia por el parto normal humanizado, teniendo conocimiento sobre el correcto abordaje de la atención profesional. Conclusión: Se concluye que la implementación del proyecto Apice On y la enfermera obstétrica contribuyeron a la reducción de casos de violencia obstétrica y cumplieron sus objetivos en vista de la humanización de partos normales realizada en el Hospital Universitario del interior de Minas Gerais.

DESCRIPTORES: Enfermera obstétrica; Parto normal; La violencia contra las mujeres.

RECEBIDO EM: 02/10/2020 APROVADO EM: 15/10/2020 


\section{Laryssa Martins Gomes}

Enfermeira. Universidade Federal de Uberlândia - UFU. Uberlândia, MG.

ORCID: 0000-0002-7605-5003

\section{Bruna Aparecida Rodrigues Duarte}

Enfermeira. Universidade Federal de Uberlândia - UFU. Uberlândia, MG.

ORCID: 0000-0003-0487-455X

\section{Carla Denari Giuliani}

Enfermeira. Mestre em Ciências Fisiológicas pela Universidade Federal de São Carlos (2001), Doutora em História e Cultura pela Universidade Federal de Uberlândia. Uberlândia, MG.

ORCID: 0000-0001-5598-2230

\section{Maria Cristina de Moura Ferreira}

Enfermeira, Doutora em Enfermagem Fundamental pela EERP-USP, Docente Associado do Curso de Graduação em Enfermagem, da Universidade Federal de Uberlândia - UFU. Uberlândia, MG.

ORCID: 0000-0002-2390-8607

\section{Marcelle Aparecida Barros Junqueira}

Enfermeira, Mestre e Doutora em Enfermagem Psiquiátrica pela Universidade de São Paulo. Uberlândia, MG.

ORCID: 0000-0002-2920-1194

\section{INTRODUÇÃO}

$\mathrm{H}$ istoricamente o parto é considerado no Brasil como um evento histórico, em que a sociedade médica se ocupa do corpo da mulher para ter lucro, permitindo que os hospitais maternidades se beneficiem financeiramente com esses partos. ${ }^{1}$ Assim, uma das grandes mudanças foi a substituição das parteiras por profissionais especializados na área da obstetrícia, essas modificações tiveram como objetivo a redução de óbitos materno/fetal. Porém, acompanhado dessas reduções tem-se a institucionalização do parto, em que "a mulher passou a ser medicalizada e a sofrer intervenções cirúrgicas que muitas vezes poderiam ser evitadas, o que hoje é reconhecido como uma das formas de violência obstétrica contra as mulheres." 1 A Violência Obstétrica (VO) pode ser conceituada como uma "expressão utilizada para descrever e agrupar diversas formas de violência (e danos) durante o cuidado obstétrico profissional." Este tipo de violência é comumente segmentada em três aspectos, são eles: VO física: ocorre quando envolvem procedimentos invasivos sem necessidade. $\mathrm{VO}$ Psíquica: quando envolvem tratamentos desrespeitosos e humilhantes, e VO sexual: ocorre quando o profissional tem acesso aos órgãos sexuais da mulher, realizando proce- dimentos que causem algum prejuízo para a parturiente. $^{3}$

Nesta perspectiva, o Ministério da Saúde - MS realizou uma pesquisa com intuito de verificar a satisfação das mulheres atendidas pelo Sistema Único de Saúde - SUS e se situações de violência obstétrica ocorriam nos serviços de saúde no ano de 2012. A amostra foi composta por 149.072 mulheres, e os resultados da pesquisa foram que $51,5 \%$ puérperas responderam que foram mal atendidas, 25,3\% puérperas não foram ouvidas, $12,1 \%$ sofreram agressão verbal, $2,4 \%$ sofreram agressão física e $8,7 \%$ puérperas sofreram outro tipo de violência. ${ }^{4}$ Com relação a taxa de cesáreas, observou-se que em 2011 $53,88 \%$ dos nascimentos ocorreram por meio de cesáreas, ${ }^{4}$ e no ano de 2019, segundo informações da Secretaria de Vigilância em Saúde (2020), a taxa de cesáreas realizadas foi de $55,86 \%$, dados que evidenciam o aumento no número de cesarianas realizadas em hospitais brasileiros. ${ }^{5}$

Tendo isso em vista, o Brasil vem esforçando-se para melhorar a assistência obstétrica em vigor e encorajar práticas com menor número de intervenções possíveis, propondo diretrizes, normas e protocolos. ${ }^{6}$ Dentro dessa ótica, foi elaborado o Projeto Apice On - Aprimoramento e Inovação no Cuidado e Ensino em Obstetrícia e Neonatologia, que busca como resultado a redução das taxas de cesariana, a realização de partos normais por enfermeiras obstétricas e ao acesso a métodos não farmacológicos para alívio da dor. ${ }^{7}$ Visando esses benefícios, o Ministério da Saúde - MS incentiva a incorporação da enfermeira obstétrica nas equipes hospitalares, tendo em vista sua contribuição para redução de possíveis intervenções e cesáreas desnecessárias. Nesse contexto, foi introduzido em um município do interior de Minas Gerais o trabalho de enfermeiras especializadas em obstetrícia para a realização dos partos ocorridos em um Hospital Universitário através do projeto Ápice On, essa inserção teve como objetivo reduzir o alto índice de cesáreas e diminuir a ocorrência de novos casos de violência obstétrica dentro do hospital. Desta forma, o objetivo deste trabalho foi analisar o quanto a implementação do projeto Apice On contribuiu para a redução de casos de violência obstétrica, vivenciadas por puérperas atendidas em um Hospital Universitário no interior de Minas Gerais.

\section{MÉTODO}

Estudo exploratório, descritivo, transversal e de caráter qualitativo, desenvolvido com puérperas atendidas em um Hospital Universitário no interior de Minas Gerais. A coleta de dados ocorreu entre os meses de Outubro e Dezembro de 2019. 
A pesquisa foi desenvolvida com puérperas de idade superior a 18 anos que estavam internadas no setor de Maternidade do Hospital no período de coleta de dados, que aceitaram participar desta pesquisa, assinaram o termo de consentimento livre e esclarecido, e responderam o questionário e entrevista. Foram excluídas do estudo as participantes que em algum momento se recusaram a participar da pesquisa e/ou a assinar o termo de consentimento livre e esclarecido, não apresentavam condições físicas e/ou psicológicas de relatarem sobre seus partos, não se encontravam em seus leitos no horário marcado para a entrevista, e puérperas que estavam amamentando no momento da coleta de dados. Com relação aos riscos oferecidos pela pesquisa, houve a possível identificação da mulher e o desconforto de relatar detalhadamente as situações ocorridas durante o trabalho de parto. Para definir o tamanho da amostra utilizaram-se dados fornecidos pelo Setor de Maternidade do Hospital através do projeto Apice On, em que foram realizados em média 86 partos vaginais entre os meses de Outubro e Dezembro de 2018. Esses dados foram inseridos em uma calculadora amostral, que teve como resultado o número 46 como tamanho da amostra, sendo necessário aplicar 46 questionários e entrevistas no setor de Maternidade do Hospital.

Em relação aos dados qualitativos, foi utilizado durante as entrevistas o gravador portátil Knup kp-8004 com intuito de manter a veracidade dos discursos, logo após a transcrição todas as gravações foram excluídas. Os dados foram coletados a partir de um instrumento semiestruturado abordando aspectos facilitadores, dificultadores e experiências vivenciadas no setor de acordo com a gestação e o período de trabalho de parto das puérperas. As perguntas presentes neste instrumento semiestruturado são: 1 - Como foi o seu processo de pré-natal?, 2 - Qual a sua visão sobre a gravidez e o trabalho de parto?, 3 - O que você sentiu no processo de gravidez e trabalho de parto?, 4 - Como foi o seu parto realizado nessa instituição?, e 5 O que você achou bom e ruim durante todo o processo de pré-natal e trabalho de parto nessa instituição?.
Para a análise de dados inicialmente foi aplicada a Análise de Conteúdo de Bardin, composta pelas seguintes etapas: organização da análise, codificação das transcrições, categorização e por último tratamento, inferência e interpretação dos resultados. $\mathrm{Na}$ etapa de categorização desta pesquisa foram criadas 13 categorias iniciais conforme os relatos mais evidentes das puérperas, 4 categorias intermediárias e posteriormente 3 categorias finais, as quais foram necessárias para a análise de dados, são elas: Assistência ao pré-natal, Influência profissional na gestação, trabalho de parto e parto e Processo de mudanças biopsicossociais. Com relação a análise qualitativa dos dados correspondentes aos grupos focais foi utilizada a técnica de análise lexical por meio do software Iramuteq (Interface de $\mathrm{R}$ pour les Analyses Multidimensionnelles de Textes et de Questionnaires), em que foi realizada inicialmente a adaptação das produções discursivas de cada entrevista às normas de preparação do corpus de análise do software Iramuteq para início da análise, resultando na Nuvem de Palavras realizada pelo software.
O projeto foi enviado ao Comitê de Ética em Pesquisa - CEP da Universidade Federal de Uberlândia seguindo todas as etapas da Resolução n. ${ }^{\circ}$ 466/12 do Conselho Nacional de Saúde. ${ }^{8}$ O projeto foi apreciado e aprovado com parecer n. ${ }^{\circ} 3.342 .104$ no CAAE: 06860619.9.0000.5152 de 2019.

\section{RESULTADOS E DISCUSSÃO}

Foram entrevistadas 46 puérperas durante a pesquisa cuja idade variou entre 18 e 37 anos. No momento da entrevista foram coletadas informações sobre a história obstétrica e idade gestacional no dia do parto. $\mathrm{O}$ quadro 1 corresponde à quantidade de puérperas de acordo com a história obstétrica, e o quadro 2 corresponde à idade gestacional das puérperas no momento do parto. Após a caracterização da amostra, foi iniciado a análise das categorias finais conforme Bardin, são elas: Assistência ao pré-natal, Influência profissional na gestação, trabalho de parto e parto, e Processos de mudanças biopsicossociais.

\section{Quadro 1 - Quantidade de puérperas de acordo com o histórico obstética}

\begin{tabular}{|l|c|c|}
\hline Gestações & Paridade & Quantidade de puérperas \\
\hline Primigestas & Primíparas & 23 \\
\hline Secundigentas & Secundíparas & 11 \\
\hline Tercigestas & Tercíparas & 5 \\
\hline Quartigestas & Quartíparas & 5 \\
\hline Multigestas & Multíparas & $\mathbf{4 6}$ \\
\hline \multicolumn{2}{|l}{} \\
\hline Fonte: Gomes; Duarte; Giuliani; Moura-Ferreira; Junqueira, 2019
\end{tabular}

Quadro 2 - Quantidade de puérperas de acordo com a idade gestacional no dia do parto

\begin{tabular}{|c|c|}
\hline Idade Gestacional & Quantidade de puérperas \\
\hline 26 semanas & 1 puérperas \\
\hline 33 semanas & 1 puérperas \\
\hline 35 semanas & 1 puérperas \\
\hline 36 semanas & 3 puérperas \\
\hline 37 semanas & 5 puérperas \\
\hline 38 semanas & 10 puérperas \\
\hline 39 semanas & 9 puérperas \\
\hline 40 semanas & 12 puérperas \\
\hline
\end{tabular}




\begin{tabular}{|c|c|}
\hline 41 semanas & 4 puérperas \\
\hline Total: & $\mathbf{4 6}$ puérperas \\
\hline Fonte: Gomes; Duarte; Giuliani; Moura-Ferreira; Junqueira, 2019 \\
\hline
\end{tabular}

\subsection{Assistência ao pré - natal}

Observou-se durante a interpretação dos dados que as puérperas possuem conhecimento sobre as recomendações do Ministério da Saúde - MS acerca de como devem ser realizadas as consultas de pré - natal, como devem ser os atendimentos feitos pelos profissionais de saúde e sobre a importância de iniciar as consultas no primeiro trimestre de gestação para que as mulheres consigam realizar todos os procedimentos necessários para cada idade gestacional. Foi percebido também que a qualidade do atendimento profissional foi um fator considerado essencial para as mulheres, em que foram transcritos inúmeros relatos de bom atendimento, humanização durante as consultas de pré natal, atenção ofertada pelo profissional durante as consultas e estímulo para a realização do parto normal, visto que todas as mulheres entrevistadas iniciaram o pré - natal com desejo de realizar o parto normal humanizado. Uma das transcrições que representa essa categoria é a fala da entrevistada Irlanda: "Eu marcava as consultas todos os meses e eles sempre me acompanhavam pesando, medindo minha barriga, medindo a pressão arterial, sempre pediam os ultrassons necessários e vários outros procedimentos. Todas as coisas que eu queria saber, conhecer sobre amamentação, sobre os acontecimentos pré e pós - parto, consegui tirar minhas dúvidas no pré - natal. Realmente dá para você tirar dúvidas e conhecer mais, tanto sobre a mãe, sobre o pós - parto e sobre o bebê. Fui bem acompanhada, não tenho o que reclamar." (Irlanda) Foi verificado também que 20 puérperas relataram que as consultas de pré - natal foram realizadas tardiamente devido à demora do Sistema Único de Saúde - SUS na marcação das consultas e exames, o que pode trazer dificuldades e impedimentos para a realização dos exames necessários para cada idade gestacional, impedindo assim, a prevenção de possíveis complicações futuras para o binômio mãe e filho.

De acordo com o preconizado pelo MS através do programa de Humanização no
Pré - Natal e Nascimento, é dever do SUS garantir que o calendário de atendimento pré - natal "seja iniciado precocemente e deve ser regular e completo garantindo-se que todas as avaliações propostas sejam realizadas." Além disso, durante a primeira consulta de pré - natal devem ser solicitados pelo profissional responsável os exames laboratoriais essenciais, como por exemplo a sorologia para Hepatite B, Sífilis e Toxoplasmose, além de realizar a atualização do cartão de vacina da gestante. A solicitação desses exames e realização de procedimentos possuem como objetivo a identificação precoce de problemas de saúde da mãe e do bebê e a prevenção de complicações futuras com ambos, sendo importante a realização dos mesmos na idade gestacional adequada. É importante ressaltar também que "o principal objetivo da atenção pré - natal e puerperal é acolher a mulher desde o início da gravidez, assegurando, no fim da gestação, o nascimento de uma criança saudável e a garantia do bem-estar materno e neonatal". ${ }^{10}$ Esse acolhimento se inicia desde a recepção até a chegada da mulher na unidade de saúde, "responsabilizando-se por ela, ouvindo suas queixas, permitindo que ela expresse suas preocupações, angústias, garantindo atenção resolutiva e articulação com os outros serviços de saúde para a continuidade da assistência, quando necessário". ${ }^{10}$ Sendo assim, apesar de algumas consultas de pré - natal terem sido realizadas fora da idade gestacional, observou-se que a maioria das puérperas foram bem atendidas, realizaram todos os procedimentos necessários e foram acolhidas de forma adequada pelos profissionais de saúde responsáveis pelo pré - natal.

\subsection{Influência profissional na gesta- ção, trabalho de parto e parto}

Nesta categoria observou-se que antes $\mathrm{da}$ realização do parto as puérperas possuíam uma visão negativa do Hospital Maternidade, ressaltando o medo de como seriam atendidas durante o trabalho de parto. Após o parto, as puérperas concluíram que o atendimento ofertado pelos profissionais foi de ótima qualidade, sendo executada de forma atenciosa, esclarecedora e humanizada, como pode ser observado no seguinte relato: "Eu tinha muito medo de vir para este hospital, de chegar aqui e falar: Será que sabem o que estão fazendo? Mas foi muito legal, os estagiários e os residentes me trataram super bem, me acompanharam o tempo todo, me deixaram tranquila, me respeitaram o tempo todo. O atendimento foi excelente." (Eslovênia) Para se ter um atendimento humanizado é extremamente importante reconhecer e respeitar a individualidade de cada mulher. $\mathrm{O}$ atendimento individual "permite ao profissional estabelecer um vínculo com cada gestante, perceber suas necessidades e capacidade de lidar com o processo do nascimento." ${ }^{11}$ Conforme o Programa de Humanização no Pré - Natal e Nascimento, além do atendimento individualizado, é dever das unidades de saúde receber com dignidade todas as mulheres, recém - nascidos e seus familiares. Para efetivar esse atendimento o profissional de saúde deve possuir atitudes éticas e acolhedoras, promovendo um ambiente hospitalar cordial e instituir rotinas que impeçam o tradicional isolamento institucional aplicado a mulher. ${ }^{12}$

Outro fator observado foi o respeito da enfermeira obstétrica quanto ao desejo pessoal da mulher para a realização de um parto humanizado, permitindo que a parturiente tivesse respeito, atenção, estímulo à realização do parto normal, acesso a métodos não farmacológicos para alívio da dor, e realização de procedimentos como episiotomia e indução somente em casos de risco para a mulher e o bebê. A seguinte transcrição representa esta categoria: "Em todos os momentos eles me deixaram à vontade, me perguntavam se eu queria mudar de posição, como eu queria ficar, como eu queria ganhar o bebê, leram o meu plano de parto, viram o que eu queria, abaixaram a iluminação e colocaram música. Eu iniciei agachada na cama, mas senti que não estava legal. Fui para a banqueta e após isso eles fizeram uma corrente de força, me puxaram com um pano enquanto eu fazia força, foi muito bom, ajudou bastante, eu gostei." (Ucrânia) "O conceito de atenção humanizada é amplo e envolve um conjunto de conhecimentos, práticas e atitudes que 
visam a promoção do parto e do nascimento saudáveis e a prevenção da morbimortalidade materna e perinatal". ${ }^{11}$ Essa atenção humanizada deve ser iniciada durante as consultas de pré - natal, e deve garantir à mulher que a equipe de saúde exerça somente procedimentos comprovadamente benéficos para o binômio mãe e filho, e que busquem evitar a realização de intervenções sem necessidade, preservando a autonomia da parturiente e sua privacidade. ${ }^{11}$ Sendo assim, os profissionais da saúde são de extrema importância durante o parto, principalmente quando se refere à humanização. Esses profissionais "podem minimizar a dor, ficar ao lado, dar conforto, esclarecer, orientar, enfim, ajudar a parir e a nascer. Precisam lembrar que são os primeiros que tocam cada ser que nasce e ter consciência dessa responsabilidade". ${ }^{11}$

Tendo essa compreensão, sabe-se que o profissional de enfermagem também tem responsabilidade na utilização dos métodos não farmacológicos para alívio da dor durante o trabalho de parto. Sabendo disso, o MS recomenda que sempre quando for possível o profissional deve oferecer a mulher a imersão em água para alívio da dor durante o trabalho de parto, apoiar técnicas de massagem e relaxamento, estimular que haja músicas durante o trabalho de parto, ensinar os movimentos realizados na bola suíça para facilitar a descida, rotação do feto e melhorar a circulação uterina, e por último estimular a deambulação da parturiente. ${ }^{13}$ Tendo em vista seus benefícios, os métodos não farmacológicos para alívio da dor foram utilizados em todos os trabalhos de partos das puérperas entrevistadas neste estudo.

\subsection{Processo de mudanças biopsicos- sociais}

Nesta categoria foi observado que a gestação altera significativamente os aspectos psicológicos, emocionais e sociais da mulher, tornando os sentimentos e sensaçôes exacerbados, como emoção, medo e ansiedade. Estes sentimentos podem também alterar a opinião das mulheres sobre a realização do parto normal e de seus procedimentos durante o parto, conforme relatado pela entrevistada Noruega: "A gestação mexe com a gente em todos os aspectos e temos que estar preparadas física e psicologicamente para as mudanças. A barriga pesa, o corpo muda, os relacionamentos com o marido e família mudam, até mesmo as nossas opiniões sobre o parto podem ser alteradas durante a gestação." (Noruega)

\section{Durante a gestação}

é comum que algumas mulheres sintam ansiedades, medos e incertezas.

Porém, "o avanço do conhecimento científico dos fenômenos físicos em obstetrícia tem proporcionado habilidades

fundamentais

a médicos e enfermeiros, permitindo-lhes

a prática de atendimento que gera, realmente, um estado de confiança maior na mulher"
Durante a gestação é comum que algumas mulheres sintam ansiedades, medos e incertezas. Porém, "o avanço do conhecimento científico dos fenômenos físicos em obstetrícia tem proporcionado habilidades fundamentais a médicos e enfermeiros, permitindo-lhes a prática de atendimento que gera, realmente, um estado de confiança maior na mulher". Entretanto, as condutas profissionais não devem ser focadas somente nos aspectos físicos de cada gestante, mas sim de forma a compreender todos os processos biopsicossociais que ocorrem durante o ciclo gravídico da mulher. Neste sentido, é importante que a enfermeira (o) ou médica (o) que atenderá a mulher, a aborde de uma forma completa, levando em consideração os sentimentos, ambientes em que a mulher vive, e sua história de vida, criando assim uma relação entre mulher/profissional que valorize a individualidade de cada pessoa. ${ }^{9}$ Neste contexto, observa-se que "os aspectos emocionais da gravidez, do parto e do puerpério são amplamente reconhecidos, e a maioria dos estudos converge para a ideia de que esse período é um tempo de grandes transformações psíquicas, de que decorre importante transição existencial." ${ }^{\prime \prime}$ Tendo isso em vista, é extremamente importante que os profissionais responsáveis por acompanhar a mulher durante a gestação e trabalho de parto estejam preparados para compreender todo esse processo de mudanças e atender a mulher com empatia e compreensão. Diante disso, é importante que as consultas de pré-natal sejam centradas na mulher e que o profissional responsável pelo pré - natal realize uma escuta acolhedora, fornecendo auxílio e permitindo que a gestante expresse seus sentimentos bons e ruins, discuta seus medos, ansiedades e dúvidas que possam surgir, para que a mulher não chegue ao trabalho de parto com esses sentimentos.

\subsection{Análise da Nuvem de Palavras}

A palavra PARTO (Figura 1) e QUERER estão em maior evidência pois se referem aos 46 partos naturais e humanizados realizados no Hospital Universitário conforme o desejo das puérperas entrevistadas. Quanto a palavra PRÉ - NATAL, foram observados que todas as puérperas realizaram 


\section{artigo}

Gomes, L.M.; Duarte B.A.R.; Giuliani, C.D.; Ferreira, M.C.M.; Junqueira, M.A.B

Violência obstétrica: perspectiva de puérperas atendidas em um hospital universitário no interior de Minas Gerais

o pré - natal, algumas tardiamente, mas as que conseguiram o realizar completamente disseram que o processo foi BOM devido a atenção dos profissionais, a realização dos exames solicitados, o alcance do objetivo de realizar todas as consultas e sobre todo o preparo do pré - natal. Outro fator observado foi que após a chegada das mulheres na Maternidade, os partos normais evoluíram de maneira RÁPIDA com a realização dos métodos não farmacológicos, e sem a necessidade de PROCEDIMENTOS como medicações desnecessárias, episiotomia e indução, e todos os processos realizados durante o parto foram explicados e informados pelos profissionais, evidenciando assim, um bom ATENDIMENTO por parte dos profissionais responsáveis.

\section{CONCLUSÃO}

Após a análise dos dados foi possível perceber que os partos realizados no Hospital Universitário ocorreram de maneira respeitosa e humanizada, com a presença de métodos não farmacológicos para alívio da dor, livre escolha da mulher frente às po-

Figura 1 - Nuvem de palavras sobre a violência obstétrica vivenciada pelas puérperas atendidas em um Hospital Universitário no período entre Agosto e Dezembro de 2019.

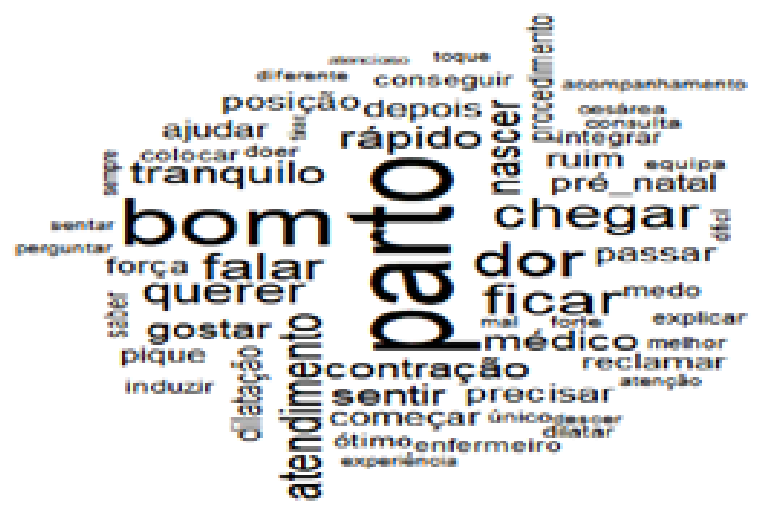

Fonte: Dados da pesquisa.

sições desejadas e técnicas de relaxamento, promovendo e permitindo o protagonismo da mulher durante a gestação e trabalho de parto, e contracenando com as recomendações do MS para humanização do parto normal, reduzindo assim situações de violência obstétrica. Sendo assim, conclui-se que a implementação do projeto Apice On e da enfermeira obstétrica contribuiu significativamente para a redução de casos de violência obstétrica e cumpriu com seus objetivos frente a humanização dos partos naturais realizados em um Hospital Universitário no interior de Minas Gerais.

\section{REFERÊNCIAS}

1. Pérez BAG, Oliveira EV, Lago MS. Percepções de puérperas vítimas de violência institucional durante o trabalho de parto e parto. REC 2015 jun; 4 (1): 66-77.

2. Tesser CD, Knobel R, Andrezzo HFA, Diniz SG. Violência obstétrica e prevenção quaternária: o que é e o que fazer. RBMFC 2015 jun; 10 (35): 1-12.

3. Pereira JS, Silva JCO, Borges NA, Ribeiro MMG, Aurek LJ, Souza JHK. Violência obstétrica: ofensa à dignidade humana. BJSCR 2016 jun; 15: 103-108.

4. Brasil. Relatório preliminar de pesquisa: resultados preliminares da pesquisa de satisfação com mulheres puérperas atendidas no Sistema Único de Saúde - SUS. Brasília (DF); 2012. [Acesso em: 11 fev. 2019]. Disponível em: https://saudenacomunidade.files.wordpress.com/2014/05/relatorio_pre_semestral_rede_cegonha_ouvidoria-sus_que-deu-a-notc3adcia-de64-por-cento-sem-acompanhantes.pdf.

5. Brasil. Painel de monitoramento de nascidos vivos segundo classificação de risco epidemiológico (Grupos de Robson). Brasília (DF); 2020. [Acesso em: 10 fev. 2020]. Disponivel em: http://svs. aids.gov.br/dantps/centrais-de-conteudos/paineis-demonitoramento/natalidade/grupos-de-robson/. Acesso em: $10 \mathrm{fev}$ 2020.
6. Souza TG, Gaiva MAM, Modes SSA. A humanização do nascimento: percepção dos profissionais de saúde que atuam na atenção ao parto. Rev Gaúcha Enferm 2011 set; 32 (3): 479-486.

7. Brasil. Apice On: Aprimoramento e inovação no cuidado e ensino em obstetrícia e neonatologia. Brasília (DF); 2018. [Acesso em: 1 jan. 2018]. Disponível em:http://portalarquivos.saude.gov. br/images/pdf/2017/agosto/18/Apice-On-2017-08-11.pdf

8. Ministério da Saúde(BR). Resolução n. ${ }^{\circ}$ 466, de 12 de dezembro de 2012. Diretrizes e normas regulamentadoras de pesquisas envolvendo seres humanos. Conselho Nacional de Saúde. Brasília, 2012.

9. Brasil. Pré-natal e puerpério: atenção qualificada e humanizada. Brasília (DF): Ministério da Saúde; 2006. Report, 3.

10. Brasil. Pré-natal e puerpério: atenção qualificada e humanizada. Brasília (DF): Ministério da Saúde; 2001. Report, 5.

11. Brasil. Parto, aborto e puerpério. Brasília (DF): Ministério da Saúde; 2001. Report, 1.

12. Brasil. Humanização do parto: humanização no pré-natal e nascimento. Brasília (DF): Ministério da Saúde; 2002. Report, 43.

13. Brasil. Diretrizes nacionais de assistência ao parto normal. Brasília (DF): Ministério da Saúde; 2017. Report, 1. 